\title{
Kinematical data on early-type galaxies. III. ${ }^{\star, \star \star}$
}

\author{
F. Simien and Ph. Prugniel \\ CRAL-Observatoire de Lyon (CNRS: UMR 142), F-69561 St-Genis-Laval Cedex, France
}

Received March 14; accepted April 17, 1997

\begin{abstract}
We present new kinematical data for a sample of 24 early-type galaxies. Rotation curves and velocitydispersion profiles are determined for 21 objects, while the central velocity dispersions are given for the whole sample. This is our third paper in a series devoted to the presentation of kinematical data on elliptical and S0 galaxies, derived from long-slit absorption spectroscopy.
\end{abstract}

Key words: galaxies: elliptical \& lenticular, cDgalaxies: kinematics and dynamics - galaxies: fundamental parameters

\section{Introduction}

We have recently begun to present kinematical measurements from absorption spectroscopy on early-type galaxies (Simien \& Prugniel 1997a,b, hereafter Papers I and II, respectively); these data are intended to contribute to the study of several structural and evolutionary issues. As part of our continued effort to get reliable velocity dispersions and rotation curves on a statistically significant sample of objects, we presently report on observations on a third list of targets.

This work follows closely the technique already described in full detail in Paper I, for both observation and reduction, and only a minimum of explanations will be given here.

\section{Sample and observations}

Our present sample consists of 24 early-type galaxies, with nine ellipticals and 15 S0s. This set adds up to the 21object and 38-object samples of Papers I and II; there are

Send offprint requests to: F. Simien (simien@obs.univ-lyon1.fr)

* Based on observations collected at the Observatoire de Haute-Provence.

** Tables 2 and 4 are presented in electronic form only; Tables 1 through 4 are available from the CDS, Strasbourg (anonymous ftp to cdsarc.u-strasbg.fr (130.79.128.5) or via http://cdsweb.u-strasbg.fr/Abstract.html) four objects in common with Paper I and two with Paper II: these additional measurements have been included in the present work for comparison purposes. Relevant catalog elements are presented in Table 1. The Es have ellipticities corresponding to classes between $\simeq \mathrm{E} 1$ and $\simeq \mathrm{E} 4$, and the S0s are moderately to highly flattened. The distances are in the range of $\simeq 10$ to $\simeq 74 \mathrm{Mpc}$ (for $\left.H_{0}=75 \mathrm{~km} \mathrm{~s}^{-1} \mathrm{Mpc}^{-1}\right)$. These objects are intrinsically bright $\left(-22.1<M_{B}<-18.9\right)$.

The observations have been secured at the $1.93-\mathrm{m}$ telescope of the Observatoire de Haute-Provence, equipped with the CARELEC long-slit spectrograph. In November 1993, November and December 1994, three observing runs totalling 10 nights collected spectra on the major axis of the galaxies and, for five of them, on the minor axis as well.

The atmospheric conditions were variable, with a seeing disk between $2^{\prime \prime}$ and $3.5^{\prime \prime}$ (FWHM) for most objects, but up to $\simeq 5^{\prime \prime}$ for three of them. The log of the observations is given in Table 2, which is proposed in electronic form only.

\section{Data reduction}

As in Papers I and II, standard pre-processing was applied to the raw data, up to the rebinning in wavelength. The galaxy centers $(r=0)$ were determined by a Gaussian fitting to a limited range $\left(\simeq 12^{\prime \prime}\right)$ around the brightest line. In the outer regions, cosmic-ray hits were removed with a median filter, and adjacent lines were combined with a variable weighting function (a Gaussian continuously wider faintward). A Fourier-Fitting technique determined the central velocity dispersion $\sigma_{0}$ and, when possible, the radial profile $\sigma(r)$ of the dispersion, together with the projected rotation curve $V(r)$ along the major axis. A two-pass mode (described in Paper I) allowed to remove cosmics on the inner lines, where the spatial resolution must be preserved. We adopted as the systemic velocity the value measured at $r=0$. Whenever possible, we have determined the maximum rotation velocity $V_{\max }$, as the mean of representative values on the opposite semi-axes. 
Table 1. Catalog elements

\begin{tabular}{|c|c|c|c|c|c|c|c|c|c|c|c|c|}
\hline Object & Type & $\alpha_{1950}$ & $\delta_{1950}$ & $v_{\text {hel }}$ & $B_{\mathrm{T}}$ & $-M_{B}$ & $r_{\mathrm{e}}$ & $\epsilon$ & $\mathrm{PA}$ & ref. & $\sigma_{0}$ & $V_{\max }$ \\
\hline$(1)$ & $(2)$ & $(3)$ & (4) & $(5)$ & $(6)$ & (7) & $(8)$ & (9) & $(10)$ & (11) & $(12)$ & $(13)$ \\
\hline NGC 0499 & -3 & 012022.4 & +331157 & 4375 & 12.96 & 20.75 & 14.9 & 0.23 & 82 & 4 & $237 \pm 24$ & $\ldots \pm \ldots$ \\
\hline NGC 0584 & -5 & 012850.2 & -070732 & 1869 & 11.29 & 20.44 & 24.5 & 0.34 & 59 & 1 & $225 \pm 12$ & $157 \pm 11$ \\
\hline NGC 0680 & -4 & 014701.4 & +214322 & 2932 & 12.39 & 20.28 & 18.5 & 0.21 & 174 & 1 & $215 \pm 14$ & $\ldots \pm \ldots$ \\
\hline NGC 0890 & -3 & $\begin{array}{lll}02 & 19 & 02.2\end{array}$ & +330217 & 4005 & 11.85 & 21.52 & 31.8 & 0.46 & 55 & 1 & $229 \pm 14$ & $<100 \pm \ldots$ \\
\hline NGC 0990 & -5 & 023336.1 & +112527 & 3508 & 13.10 & 20.00 & 18.1 & 0.19 & 43 & 1 & $176 \pm 22$ & $\ldots \pm \ldots$ \\
\hline NGC 1016 & -5 & 023544.4 & +015409 & 6583 & 12.25 & 22.09 & 51.1 & 0.09 & 59 & 1 & $280 \pm 19$ & $\ldots \pm$ \\
\hline NGC 1023 & -3 & 023715.8 & +385055 & 600 & 10.21 & 19.94 & 38.9 & 0.58 & 87 & 4 & $213 \pm 15$ & $240 \pm 36$ \\
\hline NGC 1060 & -3 & $\begin{array}{lll}02 & 40 & 13.9\end{array}$ & $+32 \quad 1247$ & 5190 & 12.06 & 21.86 & 39.5 & 0.25 & 68 & 1 & $303 \pm 29$ & $\ldots \pm \ldots$ \\
\hline NGC 1175 & -1 & $\begin{array}{lll}03 & 01 & 15.5\end{array}$ & +420843 & 5540 & 13.23 & 20.85 & 10.2 & 0.65 & 153 & 4 & $235 \pm 30$ & $160 \pm 24$ \\
\hline NGC 1209 & -5 & $\begin{array}{lll}03 & 03 & 42.9\end{array}$ & -154815 & 2617 & 12.14 & 20.15 & 23.2 & 0.46 & 82 & 1 & $241 \pm 21$ & $175 \pm 26$ \\
\hline NGC 1521 & -4 & 040608.0 & -211059 & 4172 & 12.32 & 21.22 & 34.7 & 0.22 & 19 & 1 & $236 \pm 36$ & $\ldots \pm \ldots$ \\
\hline NGC 1573 & -5 & 042903.2 & +730933 & 4220 & 12.53 & 21.03 & 22.1 & 0.31 & 35 & 4 & $286 \pm 35$ & $\ldots \pm \ldots$ \\
\hline NGC 1653 & -4 & 044316.5 & -022853 & 4338 & 12.60 & 20.92 & 25.4 & 0.08 & 118 & 1 & $253 \pm 55$ & $\ldots \pm \ldots$ \\
\hline NGC 1726 & -2 & 045716.0 & -074942 & 4008 & 12.32 & 21.20 & 29.9 & 0.29 & 7 & 1 & $236 \pm 16$ & $40 \pm 10$ \\
\hline NGC 2314 & -5 & 070353.7 & +752428 & 3848 & 12.92 & 20.51 & 10.9 & 0.12 & 25 & 4 & $290 \pm 13$ & $\ldots \pm \ldots$ \\
\hline NGC 2340 & -5 & $\begin{array}{lll}07 & 07 & 20.4\end{array}$ & +501524 & 5949 & 12.38 & 21.96 & 52.0 & 0.33 & 8 & 4 & $253 \pm 25$ & $\ldots \pm \ldots$ \\
\hline NGC 2549 & -2 & 081456.6 & +575734 & 1069 & 11.93 & 19.33 & 21.3 & 0.70 & 177 & 2 & $155 \pm 16$ & $150 \pm 30$ \\
\hline NGC 2685 & -1 & 085141.2 & +585529 & 869 & 11.94 & 19.06 & 30.1 & 0.53 & 38 & 4 & $103 \pm 17$ & $<100 \pm \ldots$ \\
\hline NGC 2732 & -2 & 090652.8 & +792333 & 1973 & 12.64 & 19.54 & 17.1 & 0.38 & 67 & 2 & $160 \pm 18$ & $129 \pm 14$ \\
\hline NGC 2768 & -4 & $\begin{array}{lll}09 & 07 & 45.2\end{array}$ & +601439 & 1334 & 10.74 & 20.86 & 61.1 & 0.68 & 95 & 3 & $193 \pm 11$ & $78 \pm 34$ \\
\hline NGC 7332 & -2 & 223501.2 & +233216 & 1202 & 11.94 & 18.98 & 11.5 & 0.55 & 156 & 1 & $136 \pm 16$ & $136 \pm 14$ \\
\hline NGC 7457 & -3 & 225836.1 & +295231 & 813 & 11.21 & 18.93 & 79.8 & 0.45 & 120 & 1 & $79 \pm 34$ & $75 \pm 11$ \\
\hline NGC 7778 & -5 & 235046.6 & +073553 & 5238 & 13.40 & 20.46 & 16.5 & 0.12 & 65 & 1 & $198 \pm 10$ & $\ldots \pm \ldots$ \\
\hline NGC 7785 & -5 & 235245.5 & +053811 & 3848 & 12.44 & 20.77 & 20.4 & 0.42 & 139 & 1 & $253 \pm 22$ & $86 \pm 25$ \\
\hline
\end{tabular}

Notes:

column (2): morphological type (from the LEDA database - status: LEDA1996);

columns (3), (4): coordinates, from Prugniel \& Simien (1997: hereafter PS97)

column (5): $v_{\text {hel }}$, heliocentric radial velocity, in $\mathrm{km} \mathrm{s}^{-1}$ (from $L E D A$ );

column (6): $B_{\mathrm{T}}$, integrated blue magnitude, corrected for Galactic extinction and $k$ term (from PS97);

column (7): $-M_{B}$, absolute $B$ magnitude (for a distance modulus from PS97, corresponding to $H_{0}=75 \mathrm{~km} \mathrm{~s}^{-1} \mathrm{Mpc}^{-1}$ );

column (8): $r_{\mathrm{e}}$, effective radius, in arcsec (from PS97);

column (9): $\epsilon$, ellipticity;

column (10): PA, position angle of major axis, in degrees (North through East, 0 to $180^{\circ}$ );

column (11): reference for $\epsilon$ and PA, $1=$ Djorgovski (1985), $2=$ Michard (1985), $3=$ Michard \& Marchal (1993), $4=L E D A$; column (12): $\sigma_{0}$, central velocity dispersion available in the literature, in $\mathrm{km} \mathrm{s}^{-1}$; from an updated version of the compilation in Prugniel \& Simien (1996: hereafter PS96) - excluding all previous measurements by ourselves;

column (13): $V_{\max }$, maximum rotation velocity available in the literature, in $\mathrm{km} \mathrm{s}^{-1}$, from PS96 (same remarks as for $\sigma_{0}$ ).

\section{Presentation of the results}

Determinations of the heliocentric radial velocity $v_{\text {hel }}$, of $\sigma_{0}$, and of $V_{\max }$ (together with the corresponding radius $\left.r_{\max }\right)$ are listed in Table 3. The $V(r)$ and $\sigma(r)$ profiles are presented in Fig. 1, and also in Table 4, which is proposed in electronic form only. Beginning with the present paper, we adopt for the position angle PA the following convention: for $0<\mathrm{PA}<180^{\circ}, r<0$ corresponds to the eastern side of the galaxy, for $180<\mathrm{PA}<360^{\circ}, r<0$ corresponds to the western side; and for PA $=0^{\circ}, r<0$ is to the North. Tables 1 through 4 are available from the CDS.

Our results are summarized as follows:
- We have measured the central velocity dispersion $\sigma_{0}$ for $24 \mathrm{E}$ and $\mathrm{S} 0$ galaxies.

- For 21 galaxies in our sample, we have been able to determine the $\sigma(r)$ and $V(r)$ profiles along the major axis and, for five of them, along the minor axis as well. For 12 of these objects, the profiles extend beyond the effective radius. Whenever possible (for 17 galaxies), we have determined the maximum rotation velocity $V_{\text {max }}$ : this parameter was still unavailable for six of these objects.

- For a few galaxies, we note secondary peaks in the velocity dispersion, which are likely the result of a strongly non-gaussian LOSVD (line-of-sight velocity distribution); the most striking example is NGC 2768. 
Table 3. Kinematical results

\begin{tabular}{lrrrr}
\hline Object & $v_{\text {hel }}$ & $\sigma_{0}$ & $V_{\max }$ & $r_{\max }$ \\
\hline$(1)$ & $(2)$ & $(3)$ & $(4)$ & $(5)$ \\
\hline $\mathrm{N} 0499$ & $4321 \pm 21$ & $284 \pm 21$ & $31 \pm 25$ & 12 \\
$\mathrm{~N} 0584$ & $1816 \pm 15$ & $185 \pm 12$ & $147 \pm 16$ & 25 \\
$\mathrm{~N} 0680$ & $2884 \pm 18$ & $191 \pm 15$ & $112 \pm 22$ & 15 \\
$\mathrm{~N} 0890$ & $3952 \pm 12$ & $212 \pm 15$ & $<32 \pm \ldots$ & 20 \\
$\mathrm{~N} 0990$ & $3515 \pm 17$ & $156 \pm 17$ & $95 \pm 32$ & 10 \\
$\mathrm{~N} 1016$ & $6567 \pm 31$ & $286 \pm 33$ & $\ldots \pm \ldots$ & $\ldots$ \\
$\mathrm{N} 1023$ & $592 \pm 15$ & $204 \pm 07$ & $205 \pm 11$ & 55 \\
$\mathrm{~N} 1060$ & $5113 \pm 51$ & $314 \pm 46$ & $\ldots \pm \ldots$ & $\ldots$ \\
$\mathrm{N} 1175$ & $5523 \pm 15$ & $225 \pm 14$ & $221 \pm 15$ & 22 \\
$\mathrm{~N} 1209$ & $2600 \pm 18$ & $227 \pm 19$ & $211 \pm 21$ & 18 \\
$\mathrm{~N} 1521$ & $4163 \pm 32$ & $224 \pm 24$ & $73 \pm 22$ & 10 \\
$\mathrm{~N} 1573$ & $4207 \pm 35$ & $306 \pm 38$ & $\ldots \pm \ldots$ & $\ldots$ \\
$\mathrm{N} 1653$ & $4343 \pm 36$ & $242 \pm 19$ & $91 \pm 38$ & 12 \\
$\mathrm{~N} 1726$ & $3995 \pm 17$ & $220 \pm 26$ & $<50 \pm \ldots$ & 20 \\
$\mathrm{~N} 2314$ & $3809 \pm 31$ & $271 \pm 19$ & $149 \pm 25$ & 15 \\
$\mathrm{~N} 2340$ & $5922 \pm 28$ & $260 \pm 30$ & $<32 \pm \ldots$ & 20 \\
$\mathrm{~N} 2549$ & $1039 \pm 10$ & $143 \pm 10$ & $160 \pm 10$ & 35 \\
$\mathrm{~N} 2685$ & $880 \pm 10$ & $92 \pm 08$ & $114 \pm 14$ & 35 \\
$\mathrm{~N} 2732$ & $1960 \pm 07$ & $149 \pm 09$ & $162 \pm 18$ & 31 \\
$\mathrm{~N} 2768$ & $1339 \pm 13$ & $176 \pm 15$ & $187 \pm 28$ & 72 \\
$\mathrm{~N} 7332$ & $1172 \pm 05$ & $131 \pm 06$ & $147 \pm 12$ & 38 \\
$\mathrm{~N} 7457$ & $812 \pm 06$ & $73 \pm 10$ & $70 \pm 14$ & 22 \\
$\mathrm{~N} 7778$ & $5278 \pm 27$ & $234 \pm 26$ & $<30 \pm \ldots$ & 15 \\
$\mathrm{~N} 7785$ & $3871 \pm 19$ & $239 \pm 19$ & $146 \pm 24$ & 22 \\
\hline & & & &
\end{tabular}

Notes:

column (2): $v_{\text {hel }}$, heliocentric radial velocity, in $\mathrm{km} \mathrm{s}^{-1}$; column (3): $\sigma_{0}$, central velocity dispersion, in $\mathrm{km} \mathrm{s}^{-1}$; column (4): $V_{\max }$, maximum rotation velocity, in $\mathrm{km} \mathrm{s}^{-1}$; column (5): $r_{\max }$, the radius at which $V_{\max }$ has been measured, in $\operatorname{arcsec}$.
Acknowledgements. We are indebted to the telescope operators at the Observatoire de Haute-Provence for their continuous support. We are pleased to thank B. Germain for his help during one of the observing runs, G. Rau for his search of the archives, and G. Busarello, the referee, for his comments. We have made use of the Lyon-Meudon Extragalactic Database supplied by the $L E D A$ team.

\section{References}

Djorgovski S., 1985, PhD thesis, Univ. of California, Berkeley Michard R., 1985, A\&AS 59, 205

Michard R., Marchal J., 1993, A\&AS 98, 29

Prugniel Ph., Simien F., 1996, A\&A 309, 749 (PS96)

Prugniel Ph., Simien F., 1997, A\&A 321, 111 (PS97)

Simien F., Prugniel Ph., 1997a, A\&AS 122, 521 (Paper I)

Simien F., Prugniel Ph., 1997b, A\&AS (in press) (Paper II) 

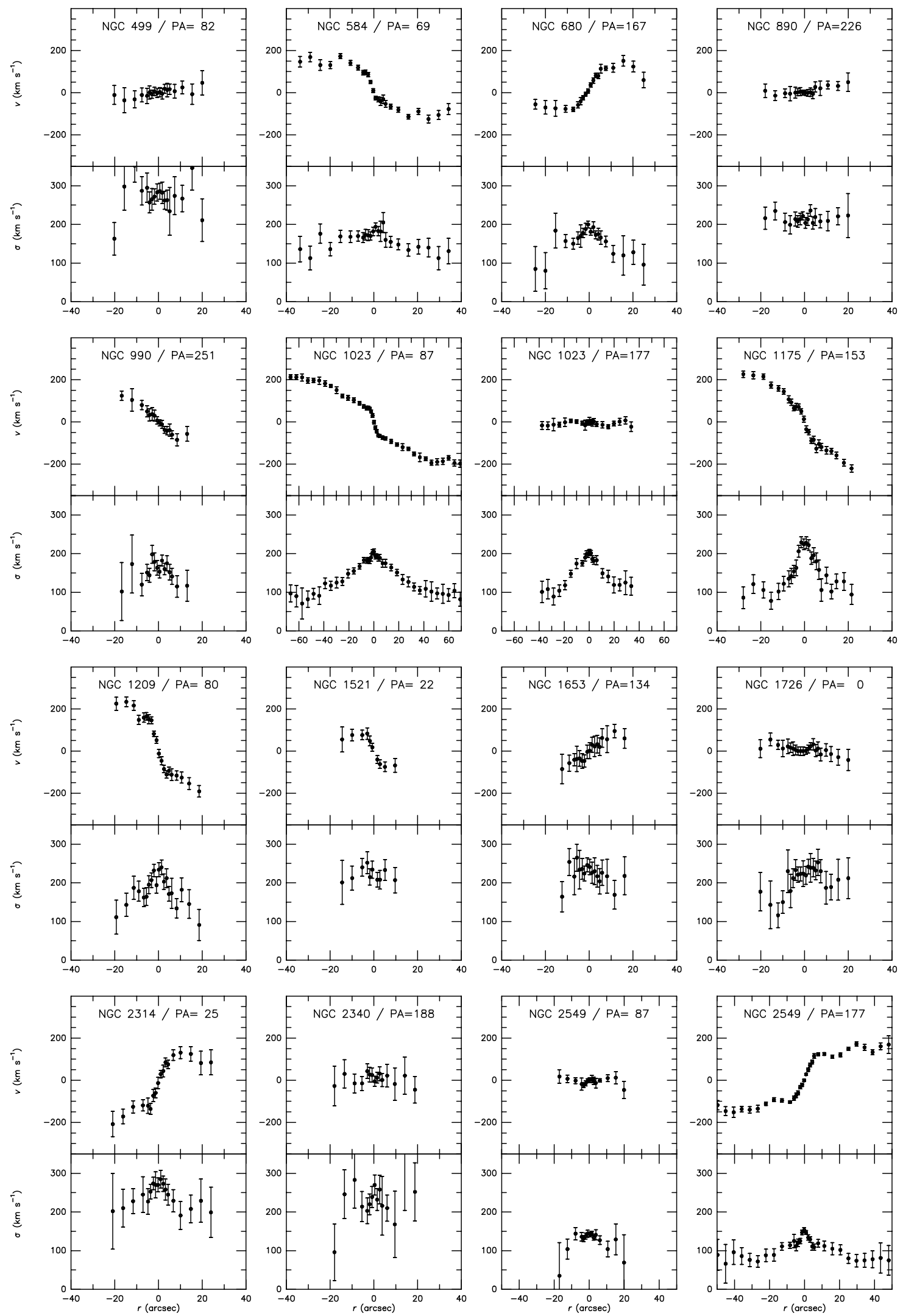

Fig. 1. Profiles of rotation velocities and velocity dispersions 

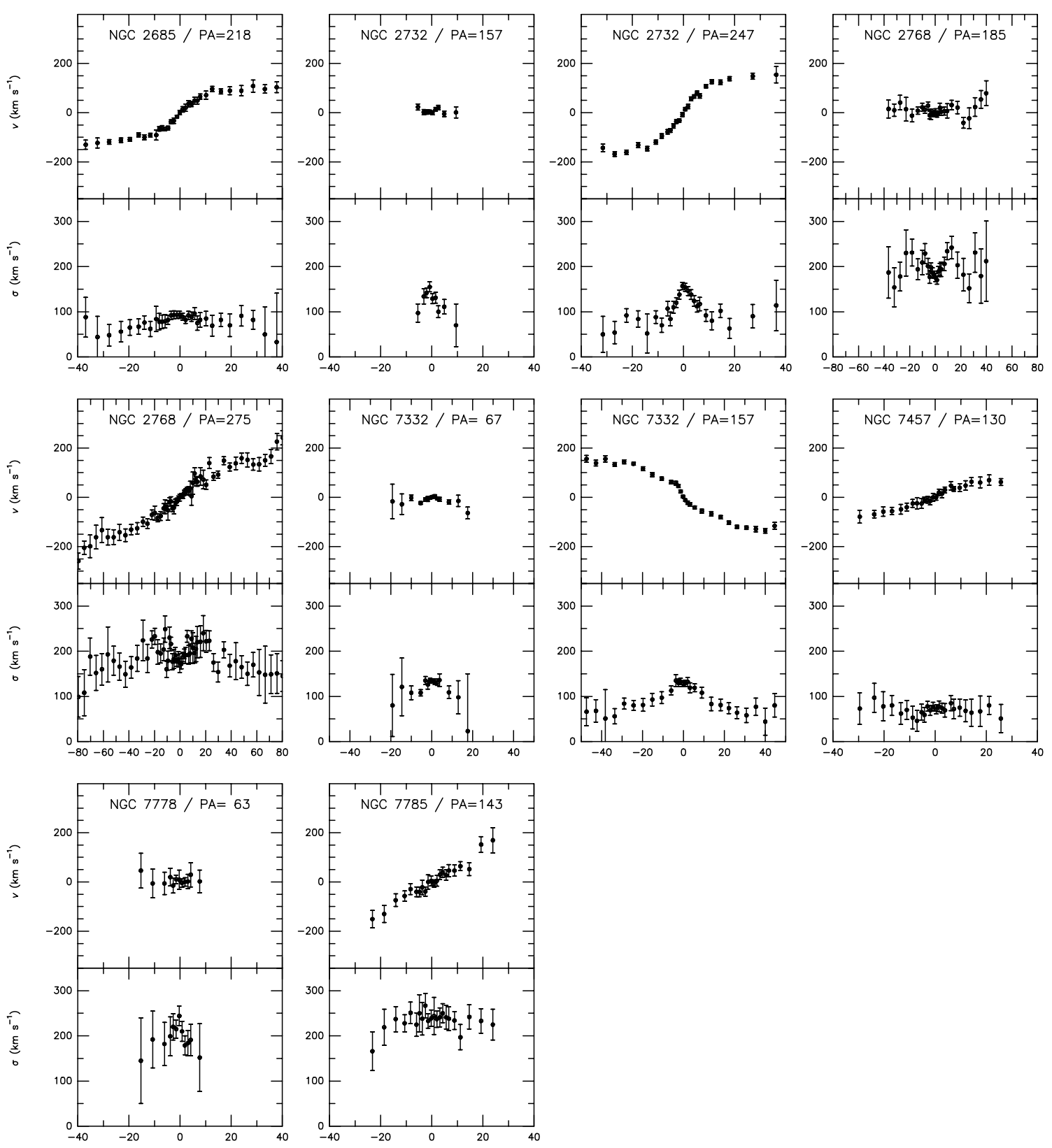

Fig. 1. continued 